\title{
Synthesis and in Vitro Antifungal Activities of Novel Triazole Antifungal Agent CS-758
}

\author{
Toshiyuki Konosu, ${ }^{*, a}$ Sadao Oida, ${ }^{a}$ Yoshie Nakamura, ${ }^{a}$ Shinobu SeKI,${ }^{a}$ Takuya UchidA, ${ }^{a}$ \\ Atsushi Somada, ${ }^{a}$ Makoto Mori,${ }^{a}$ Yoshiko Harada,${ }^{a}$ Yasuki Kamai,${ }^{b}$ Tamako Harasaki, ${ }^{b}$ \\ Takashi Fukuoka, ${ }^{b}$ Satoshi Ohya, ${ }^{b}$ Hiroshi Yasuda,,${ }^{b}$ Takahiro Shibayama, ${ }^{c}$ Shin-ichi Inoue, ${ }^{c}$ \\ Akihiko NaKagawa, ${ }^{c}$ and Yasuo SeTA ${ }^{d}$ \\ Medicinal Chemistry Research Laboratories, ${ }^{a}$ Biological Research Laboratories, ${ }^{b}$ Pharmacokinetics and Drug Delivery \\ Research Laboratories, ${ }^{c}$ and Product Development Laboratories, ${ }^{d}$ Sankyo Co., Ltd., 1-2-58 Hiromachi, Shinagawa-ku, \\ Tokyo 140-8710, Japan. Received July 9, 2001; accepted September 17, 2001
}

\begin{abstract}
Synthesis and in vitro antifungal activities of a novel triazole antifungal agent CS-758 (former name, R120758) are described. The minimum inhibitory concentrations (MICs) of a series of dioxane-triazole compounds related to R-102557 were examined. Variation of the length of the chain between the dioxane ring and the phenyl ring revealed that the linkage with two double bonds is the most preferable. When a cyano group was introduced to the $\mathrm{C} 4$ position on the benzene ring, MICs improved further. A fluorine atom was introduced to obtain CS-758. The MICs of CS-758 surpassed those of fluconazole and itraconazole against Candida, Aspergillus and Cryptococcus species. The precursor $(E, E)$-aldehyde was synthesized stereoselectively from 3-fluoro-4methylbenzonitrile using the Horner-Wadsworth-Emmons reaction.
\end{abstract}

Key words CS-758; R-120758; antifungal; triazole; Horner-Wadsworth-Emmons reaction

Recently we synthesized a series of dioxane-triazole antifungal agents, which are effective against systemic mycoses. ${ }^{1)}$ From such compounds, R-102557 (3d) was chosen as a candidate compound for development on the basis of its in vivo activities in mice. Since it was found that R-102557's high in vivo activities were caused by its excessively long elimination half-life in plasma, ${ }^{2)}$ we determined to search for a similar compound but with a shorter half-life. In this paper, we will describe the synthesis and in vitro antifungal activities of our new compound CS-758 (3j; former name, R120758), ${ }^{3)}$ which is currently under development as an antifungal agent against systemic mycoses.

Starting our search, our attention was focused on minimun inhibitory concentrations (MICs) because as the elimination half-life in plasma gets shorter, the requirements for MICs become severer. We reasoned that, even if a compound with good MICs were to exhibit only fair in vivo activities in mice, the compound might still be able to remain sufficiently

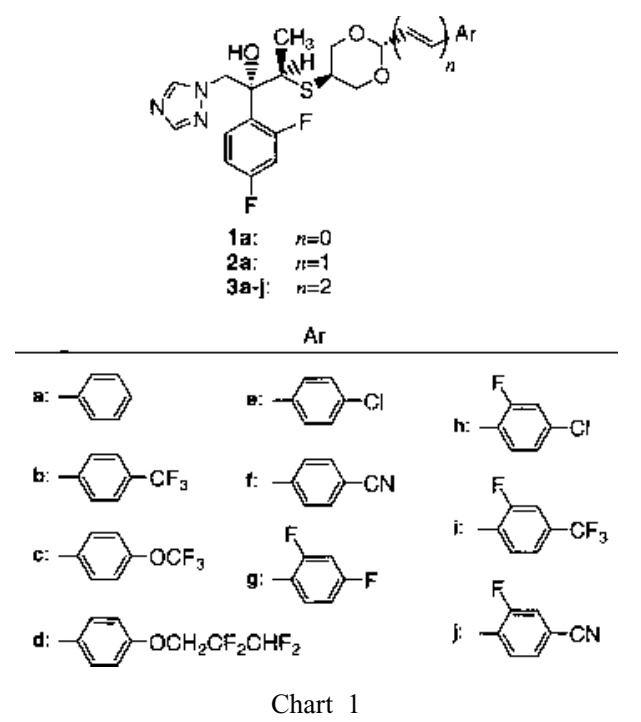

long in humans to exhibit reasonable therapeutic effects. On this assumption, some of the previous compounds, such as $\mathbf{3 b}-\mathbf{g}{ }^{1)}$ were re-evaluated on the basis of MICs. Their precursor 1a was also submitted for MIC determination. Some additional compounds, such as $\mathbf{2 a}, \mathbf{3 a}, \mathbf{3 h}-\mathbf{j}$, were prepared.

Preparation of the compounds $\mathbf{2 a}, \mathbf{3 a}, \mathbf{3} \mathbf{h}-\mathbf{j}$ were done in acetalization reactions of $\mathbf{4}$ with aldehydes $5 \mathbf{a}, 6 \mathbf{6}, \mathbf{6 h}-\mathbf{j}$, respectively (Chart 2). Previously, molecular sieves were then used to remove water, ${ }^{1)}$ but this time we conducted the reaction by concentrating a tetrahydrofuran (THF) solution containing 4 , the aldehydes, and $p$-toluenesulfonic acid in a rotary evaporator. Aldehyde $\mathbf{6 j}$, a precursor of CS-758, was

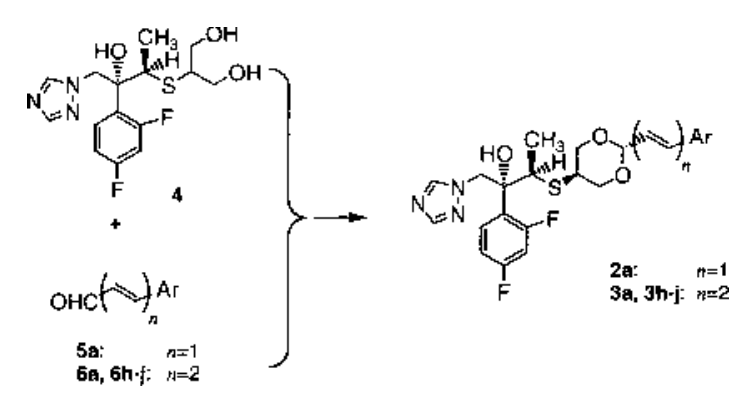

Chart 2

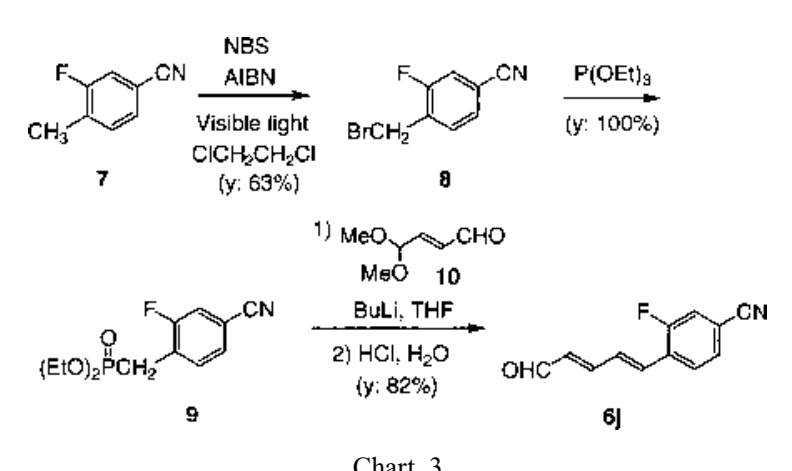

Chart 3 
prepared stereoselectively using the Horner-Wadsworth-Emmons reaction of fumaraldehyde monoacetal $\mathbf{1 0}$ as depicted in Chart 3.

The MICs of the compounds with various side chain lengths were determined against clinically important pathogenic fungi (Table 1). An unsubstituted phenyl group was first selected as the Ar group and compounds 1a $(n=0), 2 \mathbf{a}$ $(n=1)$, and 3a $(n=2)$ were compared. Compound 3a with two olefinic double bonds $(n=2)$ showed the best MICs. ${ }^{4}$
The difference was most clear in the activity against Cryptococcus neoformans.

We then fixed $n$ to 2, and compared the MICs of the derivatives $\mathbf{3 b}-\mathbf{f}$ having various substituents at the $\mathrm{C} 4$ position on the benzene ring Ar (Table 2). The compound with a cyano group (3f) showed remarkable effect with excellent MICs. The difference was the most clear in the activity against $A s$ pergillus fumigatus.

We finally introduced a fluorine atom to the $\mathrm{C} 2$ position on

Table 1. In Vitro Antifungal Activities of Compounds 1a, 2a, 3a

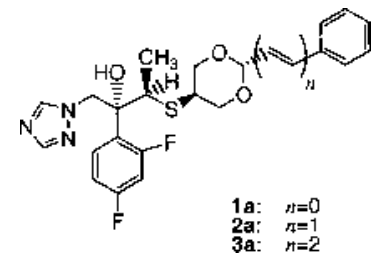

\begin{tabular}{|c|c|c|c|c|c|}
\hline \multirow[b]{2}{*}{ Compound } & \multirow[b]{2}{*}{$n$} & \multicolumn{4}{|c|}{$\mathrm{MIC}(\mu \mathrm{g} / \mathrm{ml})$} \\
\hline & & $\begin{array}{l}\text { Candida albicans } \\
\text { SANK } 51486\end{array}$ & $\begin{array}{c}\text { Cryptococcus neoformans } \\
\text { TIMM } 1855\end{array}$ & $\begin{array}{l}\text { Aspergillus fumigatus } \\
\text { SANK } 10569\end{array}$ & $\begin{array}{l}\text { Aspergillus flavus } \\
\text { SANK } 18497\end{array}$ \\
\hline $1 \mathrm{a}$ & 0 & $\leqq 0.008$ & 0.25 & 4 & $>4$ \\
\hline $2 a$ & 1 & $\leqq 0.008$ & 0.031 & 0.25 & 2 \\
\hline 3a & 2 & $\leqq 0.008$ & 0.016 & 0.25 & 1 \\
\hline
\end{tabular}

Table 2. In Vitro Antifungal Activites of Compounds $\mathbf{3 a}-\mathbf{j}$

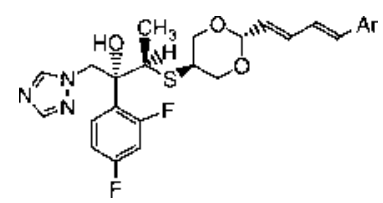

\begin{tabular}{|c|c|c|c|c|c|}
\hline \multirow[b]{2}{*}{ Compound } & \multirow[b]{2}{*}{$\mathrm{Ar}$} & \multicolumn{4}{|c|}{$\operatorname{MIC}(\mu \mathrm{g} / \mathrm{ml})$} \\
\hline & & $\begin{array}{l}\text { Candida albicans } \\
\text { SANK } 51486\end{array}$ & $\begin{array}{c}\text { Cryptococcus neoformans } \\
\text { TIMM } 1855\end{array}$ & $\begin{array}{l}\text { Aspergillus fumigatus } \\
\text { SANK } 10569\end{array}$ & $\begin{array}{l}\text { Aspergillus flavus } \\
\text { SANK } 18497\end{array}$ \\
\hline $3 \mathbf{a}$ & & $\leqq 0.008$ & 0.016 & 0.25 & 1 \\
\hline $3 \mathbf{b}$ & & 0.016 & 0.016 & 0.25 & 2 \\
\hline $3 c$ & & 0.031 & 0.031 & 0.5 & 1 \\
\hline $\begin{array}{c}\text { 3d } \\
(\mathrm{R}-102557)\end{array}$ & & 0.016 & 0.031 & 0.125 & 0.25 \\
\hline $3 \mathbf{e}$ & & $\leqq 0.008$ & 0.016 & 0.125 & 0.25 \\
\hline $3 f$ & & $\leqq 0.008$ & 0.016 & 0.063 & 0.25 \\
\hline $3 g$ & & 0.016 & $\leqq 0.008$ & 0.25 & 0.5 \\
\hline $3 h$ & & 0.016 & 0.016 & 0.25 & 0.5 \\
\hline $3 \mathbf{i}$ & & 0.016 & 0.031 & 0.25 & 1 \\
\hline $\begin{array}{c}\mathbf{3 j} \\
(\mathrm{CS}-758)\end{array}$ & & $\leqq 0.008$ & 0.016 & 0.063 & 0.25 \\
\hline
\end{tabular}


Table 3. In Vitro Antifungal Activites of CS-758, Fluconazole, and Itraconazole

\begin{tabular}{lccc}
\hline \hline \multicolumn{1}{c}{ Strain } & \multicolumn{3}{c}{ MIC $(\mu \mathrm{g} / \mathrm{ml})$} \\
\cline { 2 - 4 } & CS-758 & Fluconazole & Itraconazole \\
\hline C. albicans ATCC 24433 & 0.016 & 0.5 & 0.125 \\
C. albicans SANK 51486 & $\leqq 0.008$ & 0.25 & 0.031 \\
C. albicans TIMM 3164 & 0.063 & $>4$ & 0.25 \\
C. albicans ATCC 64550 & 0.5 & $>4$ & 1 \\
C. parapsilosis ATCC 90018 & 0.016 & 0.5 & 0.125 \\
C. glabrata ATCC 90030 & 1 & $>4$ & 1 \\
C. krusei ATCC 6258 & 0.25 & $>4$ & 0.5 \\
C. tropicalis ATCC 750 & 0.25 & 2 & 0.5 \\
C. neoformans TIMM 1855 & 0.016 & $>4$ & 0.25 \\
A. fumigatus ATCC 26430 & 0.063 & $>4$ & 0.25 \\
A. fumigatus SANK 10569 & 0.063 & $>4$ & 0.25 \\
A. flavus SANK 18497 & 0.25 & $>4$ & 0.5 \\
\hline
\end{tabular}

the benzene ring to obtain CS-758 (3j). The aim of the fluorine atom was to improve the solubility and chemical/metabolic stability. ${ }^{5)} \mathrm{CS}-758(\mathbf{3 j})$ showed comparable MICs to those of 3f. Analogs 3g-i, with a fluorine atom at the C2 position but without a cyano group at the $\mathrm{C} 4$ position, showed lower activities. The excellent MIC of $\mathbf{3 g}$ against Cryptococcus neoformans was an exception.

The MICs of CS-758, fluconazole, and itraconazole against 12 fungal strains are listed in Table 3 . The MICs of CS-758 were excellent and surpassed those of the reference drugs. Further evaluations are under way.

\section{Experimental}

Melting points were determined with a Yanagimoto micro melting point apparatus and are uncorrected. IR spectra were recorded on a Nic 5SCX spectrometer. ${ }^{1} \mathrm{H}-\mathrm{NMR}$ spectra were recorded either on a JEOL GX-270 $(270 \mathrm{MHz})$, on a Varian Mercury $400(400 \mathrm{MHz})$, or on a JNM GSX-400 $(400 \mathrm{MHz})$ spectrometer using tetramethylsilane as an internal standard. The abbreviations are as follows: s, singlet; d, doublet; dd, doublet of doublets; ddd, doublet of doublets of doublets; t, triplet; dt, doublet of triplets; td, triplet of doublets; tt, triplet of triplets; q, quartet; m, multiplet; br, broad. MS and high-resolution MS (HR-MS) were recorded either on a JEOL JMS D300, on a JEOL BU30, or on a JEOL JMS-700 spectrometer. Column chromatography was carried out on silica gel (Kieselgel 60, 0.040-0.063 $\mathrm{mm}$, Merck). The amounts of silica gel used are shown in parentheses.

$(2 R, 3 R)$-2-(2,4-Difluorophenyl)-3-[[trans-2-[(E)-styryl]-1,3-dioxan-5yl]thio]-1-(1H-1,2,4-triazol-1-yl)-2-butanol (2a) and $(2 R, 3 R)-2-(2,4-D i-$ fluorophenyl)-3-[[trans-2-[(2E,4E)-5-aryl-2,4-pentadienyl]-1,3-dioxan-5yl]thio]-1-(1 H-1,2,4-triazol-1-yl)-2-butanols (3a, 3h, 3i) As a typical example, the preparation of $\mathbf{2 a}$ is described. A solution of $(2 R, 3 R)-2-(2,4-d i f l u-$ orophenyl)-3-[[2-hydroxy-1-(hydroxymethyl)ethyl]thio]-1-(1H-1,2,4-triazol1-yl)-2-butanol (4; $1.10 \mathrm{~g}, 2.78 \mathrm{mmol}){ }^{1)}(E)$-cinnamaldehyde $(5 \mathrm{a} ; 404 \mathrm{mg}$, $3.06 \mathrm{mmol})$, and $p$-toluenesulfonic acid monohydrate $(581 \mathrm{mg}, 3.05 \mathrm{mmol})$ in THF $(10 \mathrm{ml})$ was allowed to stand at room temperature for $5 \mathrm{~min}$, and then concentrated in vacuo using a rotary evaporator to remove water azeotropically. Similar processes (addition of THF and concentration) were repeated two more times. The residual oil was dissolved in THF and cautiously poured into a stirred aqueous solution of $\mathrm{NaHCO}_{3}$ at $0{ }^{\circ} \mathrm{C}$. The mixture was partitioned between EtOAc and water. The organic layer was washed with brine and dried over $\mathrm{MgSO}_{4}$. Evaporation of the solvent afforded an oily residue, which was chromatographed on silica gel $(60 \mathrm{~g})$. Elution with a mixture of EtOAc and hexane $(1: 1$ then $4: 1, \mathrm{v} / \mathrm{v})$ afforded 2a $(796.5 \mathrm{mg}$, $61 \%)$ as a colorless, amorphous solid. $[\alpha]_{\mathrm{D}}^{25}-86.1^{\circ}\left(c=1.64, \mathrm{CHCl}_{3}\right)$. IR $(\mathrm{KBr}) \mathrm{cm}^{-1}: 1617,1499,1388,1274,1137,1050,966,852 .{ }^{1} \mathrm{H}-\mathrm{NMR}(400$ $\left.\mathrm{MHz}, \mathrm{CDCl}_{3}\right) \delta: 1.19(3 \mathrm{H}, \mathrm{d}, J=7 \mathrm{~Hz}), 3.34(1 \mathrm{H}, \mathrm{q}, J=7 \mathrm{~Hz}), 3.42(1 \mathrm{H}, \mathrm{tt}$, $J=11,5 \mathrm{~Hz}), 3.65(1 \mathrm{H}, \mathrm{t}, J=11 \mathrm{~Hz}), 3.67(1 \mathrm{H}, \mathrm{t}, J=11 \mathrm{~Hz}), 4.35(1 \mathrm{H}, \mathrm{ddd}$, $J=11,5,2 \mathrm{~Hz}), 4.44(1 \mathrm{H}, \mathrm{ddd}, J=11,5,2 \mathrm{~Hz}), 4.83(1 \mathrm{H}, \mathrm{d}, J=14 \mathrm{~Hz}), 5.02$ $(1 \mathrm{H}, \mathrm{s}, J=14 \mathrm{~Hz}), 5.04(1 \mathrm{H}, \mathrm{d}, J=14 \mathrm{~Hz}), 5.13(1 \mathrm{H}, \mathrm{d}, J=5 \mathrm{~Hz}), 6.18(1 \mathrm{H}$, dd, $J=16,5 \mathrm{~Hz}), 6.7-6.8(2 \mathrm{H}, \mathrm{m}), 6.80(1 \mathrm{H}, \mathrm{d}, J=16 \mathrm{~Hz}), 7.25-7.42(6 \mathrm{H}, \mathrm{m})$, $7.79(2 \mathrm{H}, \mathrm{s})$. HR-MS (FAB) $m / z$ : Calcd for $\mathrm{C}_{24} \mathrm{H}_{26} \mathrm{~F}_{2} \mathrm{~N}_{3} \mathrm{O}_{3} \mathrm{~S}(\mathrm{M}+\mathrm{H})^{+}$:
474.1663. Found: 474.1672.

Compounds $\mathbf{3 a}, \mathbf{3 h}$, and $\mathbf{3 i}$ were prepared similarly from aldehydes $\mathbf{6 a}$, $\mathbf{6 h}$, and $\mathbf{6 i}$, respectively.

3a: Amorphous solid, $69 \% .[\alpha]_{\mathrm{D}}^{25}-80.3^{\circ}\left(c=1.13, \mathrm{CHCl}_{3}\right) .{ }^{1} \mathrm{H}-\mathrm{NMR}$ $\left(400 \mathrm{MHz}, \mathrm{CDCl}_{3}\right) \delta: 1.18(3 \mathrm{H}, \mathrm{d}), 3.33(1 \mathrm{H}, \mathrm{q}, J=7 \mathrm{~Hz}), 3.39(1 \mathrm{H}, \mathrm{tt}$, $J=11,5 \mathrm{~Hz}), 3.62(1 \mathrm{H}, \mathrm{t}, J=11 \mathrm{~Hz}), 3.64(1 \mathrm{H}, \mathrm{t}, J=11 \mathrm{~Hz}), 4.30(1 \mathrm{H}, \mathrm{ddd}$, $J=11,4,2 \mathrm{~Hz}), 4.41(1 \mathrm{H}, \mathrm{ddd}, J=11,4,2 \mathrm{~Hz}), 4.83(1 \mathrm{H}, \mathrm{d}, J=14 \mathrm{~Hz}), 5.00$ $(1 \mathrm{H}, \mathrm{d}, J=1 \mathrm{~Hz}), 5.03(1 \mathrm{H}, \mathrm{d}, J=14 \mathrm{~Hz}), 5.05(1 \mathrm{H}, \mathrm{d}, J=4 \mathrm{~Hz}), 5.77(1 \mathrm{H}, \mathrm{dd}$, $J=15,4 \mathrm{~Hz}), 6.59(1 \mathrm{H}, \mathrm{dd}, J=15,11 \mathrm{~Hz}), 6.63(1 \mathrm{H}, \mathrm{d}, J=15 \mathrm{~Hz}), 6.7-6.8$ $(2 \mathrm{H}, \mathrm{m}), 6.78(1 \mathrm{H}, \mathrm{dd}, J=15,11 \mathrm{~Hz}), 7.20-7.25(1 \mathrm{H}, \mathrm{m}), 7.3-7.4(3 \mathrm{H}, \mathrm{m})$, $7.41(2 \mathrm{H}, \mathrm{d}, J=5 \mathrm{~Hz}), 7.79(2 \mathrm{H}, \mathrm{s})$. IR $(\mathrm{KBr}) \mathrm{cm}^{-1}: 1617,1597,1499,1450$, $1420,1388,1275,1140,1050,966,899,852$. HR-MS (FAB) $m / z$ : Calcd for $\mathrm{C}_{26} \mathrm{H}_{28} \mathrm{~F}_{2} \mathrm{~N}_{3} \mathrm{O}_{3} \mathrm{~S}(\mathrm{M}+\mathrm{H})^{+}:$500.1819. Found: 500.1814 .

3h: Amorphous solid, $83 \%$. $[\alpha]_{\mathrm{D}}^{25}-73.6^{\circ}\left(c=0.96, \mathrm{CHCl}_{3}\right)$. IR $(\mathrm{KBr})$ $\mathrm{cm}^{-1}: 1617,1602,1499,1488,1416,1388,1275,1141,1050,967,900$, 854. ${ }^{1} \mathrm{H}-\mathrm{NMR}\left(400 \mathrm{MHz}, \mathrm{CDCl}_{3}\right) \delta: 1.18(3 \mathrm{H}, \mathrm{d}, J=7 \mathrm{~Hz}), 3.33(1 \mathrm{H}, \mathrm{q}$, $J=7 \mathrm{~Hz}), 3.39(1 \mathrm{H}, \mathrm{tt}, J=11,5 \mathrm{~Hz}), 3.62(1 \mathrm{H}, \mathrm{t}, J=11 \mathrm{~Hz}), 3.64(1 \mathrm{H}, \mathrm{t}$, $J=11 \mathrm{~Hz}), 4.30(1 \mathrm{H}$, ddd, $J=11,5,2 \mathrm{~Hz}), 4.41(1 \mathrm{H}, \mathrm{ddd}, J=11,5,2 \mathrm{~Hz})$, $4.83(1 \mathrm{H}, \mathrm{d}, J=14 \mathrm{~Hz}), 5.01(1 \mathrm{H}, \mathrm{s}), 5.03(1 \mathrm{H}, \mathrm{d}, J=14 \mathrm{~Hz}), 5.05(1 \mathrm{H}, \mathrm{d}$, $J=4 \mathrm{~Hz}), 5.81(1 \mathrm{H}, \mathrm{dd}, J=15,4 \mathrm{~Hz}), 6.58(1 \mathrm{H}, \mathrm{dd}, J=15,11 \mathrm{~Hz}), 6.7-6.8$ $(2 \mathrm{H}, \mathrm{m}), 6.70(1 \mathrm{H}, \mathrm{d}, J=15 \mathrm{~Hz}), 6.82(1 \mathrm{H}, \mathrm{dd}, J=15,11 \mathrm{~Hz}), 7.05-7.12$ $(2 \mathrm{H}, \mathrm{m}), 7.3-7.45(2 \mathrm{H}, \mathrm{m}), 7.79(2 \mathrm{H}, \mathrm{s})$. HR-MS (FAB) $\mathrm{m} / \mathrm{z}$ : Calcd for $\mathrm{C}_{26} \mathrm{H}_{26} \mathrm{ClF}_{3} \mathrm{~N}_{3} \mathrm{O}_{3} \mathrm{~S}(\mathrm{M}+\mathrm{H})^{+}:$552.1335. Found: 552.1328 .

3i: Amorphous solid, $63 \%$. $[\alpha]_{\mathrm{D}}^{25}-70.3^{\circ}\left(c=1.00, \mathrm{CHCl}_{3}\right)$. IR $(\mathrm{KBr})$ $\mathrm{cm}^{-1}: 1618,1500,1429,1331,1276,1171,1138,1050,993 .{ }^{1} \mathrm{H}-\mathrm{NMR}$ $\left(400 \mathrm{MHz}, \mathrm{CDCl}_{3}\right) \delta: 1.19(3 \mathrm{H}, \mathrm{d}, J=7 \mathrm{~Hz}), 3.33(1 \mathrm{H}, \mathrm{q}, J=7 \mathrm{~Hz}), 3.40(1 \mathrm{H}$, $\mathrm{tt}, J=11,5 \mathrm{~Hz}), 3.62(1 \mathrm{H}, \mathrm{t}, J=11 \mathrm{~Hz}), 3.64(1 \mathrm{H}, \mathrm{t}, J=11 \mathrm{~Hz}), 4.31(1 \mathrm{H}$, ddd, $J=11,5,2 \mathrm{~Hz}), 4.42(1 \mathrm{H}$, ddd, $J=11,5,2 \mathrm{~Hz}), 4.83(1 \mathrm{H}, \mathrm{d}, J=14 \mathrm{~Hz})$, $5.00(1 \mathrm{H}, \mathrm{s}), 5.03(1 \mathrm{H}, \mathrm{d}, J=14 \mathrm{~Hz}), 5.07(1 \mathrm{H}, \mathrm{d}, J=4 \mathrm{~Hz}), 5.87(1 \mathrm{H}, \mathrm{dd}$, $J=15,4 \mathrm{~Hz}), 6.62(1 \mathrm{H}, \mathrm{dd}, J=15,11 \mathrm{~Hz}), 6.7-6.8(2 \mathrm{H}, \mathrm{m}), 6.77(1 \mathrm{H}, \mathrm{d}$, $J=16 \mathrm{~Hz}), 6.93(1 \mathrm{H}, \mathrm{dd}, J=16,11 \mathrm{~Hz}), 7.31(1 \mathrm{H}, \mathrm{d}, J=10 \mathrm{~Hz}), 7.33-7.39$ $(2 \mathrm{H}, \mathrm{m}), 7.59(1 \mathrm{H}, \mathrm{t}, J=8 \mathrm{~Hz}), 7.79(2 \mathrm{H}, \mathrm{s})$. HR-MS (FAB) $\mathrm{m} / z$ : Calcd for $\mathrm{C}_{27} \mathrm{H}_{26} \mathrm{~F}_{6} \mathrm{~N}_{3} \mathrm{O}_{3} \mathrm{~S}(\mathrm{M}+\mathrm{H})^{+}:$586.1599. Found: 586.1602 .

4-(Bromomethyl)-3-fluorobenzonitrile (8) ${ }^{6}$ In a $2000 \mathrm{ml}$ round-bottomed Pyrex glass flask fitted with a condenser, a mixture of 3-fluoro-4methylbenzonitrile (7;96 g, $710 \mathrm{mmol}), \mathrm{N}$-bromosuccinimide (NBS; $126 \mathrm{~g}$, $708 \mathrm{mmol}$ ), 2,2'-azobis(isobutyronitrile) (AIBN; $1.2 \mathrm{~g}, 7 \mathrm{mmol}$ ) and 1,2dichloroethane $(1000 \mathrm{ml})$ was stirred with a magnetic stirrer and irradiated with a tungsten lamp $(375 \mathrm{~W})$. The mixture was allowed to warm by the light of the lamp. When the mixture started to reflux, the lamp was occasionally turned off to prevent violent refluxing. After $20 \mathrm{~min}$ of refluxing, the redbrown color of the bromine disappeared and the solution became yellow. The mixture was irradiated for $5 \mathrm{~min}$ further, and then allowed to cool to room temperature. The mixture was then cooled with ice, and the precipitated succinimide was removed by filtration. The filtrate was partitioned between EtOAc and $10 \%(\mathrm{w} / \mathrm{w}) \mathrm{NaCl}$. The organic layer was dried over $\mathrm{MgSO}_{4}$ and solvents were removed in vacuo to afford a crude solid, which was recrystallized from hexane-EtOAc to afford 8 (96 g, 63\% yield) as colorless needles, mp $71-74^{\circ} \mathrm{C}$. Anal. Calcd for $\mathrm{C}_{8} \mathrm{H}_{5} \mathrm{BrFN}$ : C, 44.89; $\mathrm{H}$, 2.36; N, 6.54. Found: C, 45.01; H, 2.36; N, 6.67. IR (KBr) $\mathrm{cm}^{-1}: 2238$, $1573,1502,1417,1264,954,895,841 .{ }^{1} \mathrm{H}-\mathrm{NMR}\left(270 \mathrm{MHz}, \mathrm{CDCl}_{3}\right) \delta: 4.49$ $(2 \mathrm{H}, \mathrm{s}), 7.38(1 \mathrm{H}, \mathrm{dd}, J=9,1 \mathrm{~Hz}), 7.45(1 \mathrm{H}, \mathrm{dd}, J=8,1 \mathrm{~Hz}), 7.54(1 \mathrm{H}, \mathrm{t}$, $J=8 \mathrm{~Hz}$ ). MS [electron impact (EI)] $m / z: 215,213\left(\mathrm{M}^{+}\right), 134(100 \%), 107$. HR-MS (EI) $m / z$ : Calcd for $\mathrm{C}_{8} \mathrm{H}_{5} \mathrm{BrFN}\left(\mathrm{M}^{+}\right)$: 214.9569. Found: 214.9542.

Diethyl 4-Cyano-2-fluorobenzylphosphonate (9) A mixture of 8 (1.5 $\mathrm{g}, 7.0 \mathrm{mmol})$ and triethyl phosphite $(1.4 \mathrm{~g}, 8.4 \mathrm{mmol})$ was heated at $150^{\circ} \mathrm{C}$ for $2 \mathrm{~h}$. The mixture was then concentrated under reduced pressure, and the residue was heated at $100^{\circ} \mathrm{C}$ while being suctioned with a vacuum pump to remove volatile materials. The crude oil thus obtained $(1.97 \mathrm{~g}$, quantitative yield) was used for the next reaction without further purification. IR $\left(\mathrm{CHCl}_{3}\right)$ $\mathrm{cm}^{-1}: 2237,1262,1054,1029 .{ }^{1} \mathrm{H}-\mathrm{NMR}\left(270 \mathrm{MHz}, \mathrm{CDCl}_{3}\right) \delta: 1.27(6 \mathrm{H}, \mathrm{t}$, $J=7.1 \mathrm{~Hz}), 3.24(2 \mathrm{H}, \mathrm{d}, J=22.3 \mathrm{~Hz}), 4.00-4.05(4 \mathrm{H}, \mathrm{m}), 7.37(1 \mathrm{H}, \mathrm{d}$, $J=9.2 \mathrm{~Hz}), 7.43(1 \mathrm{H}, \mathrm{d}, J=7.9 \mathrm{~Hz}), 7.51(1 \mathrm{H}, \mathrm{td}, J=9.2,2.6 \mathrm{~Hz})$. MS (EI) $m / z: 271\left(\mathrm{M}^{+}\right) 139,109,93$.

3-Fluoro-4-[(1E,3E)-5-0xo-1,3-pentadienyl]benzonitrile (6j) A solution of butyllithium $(1.53 \mathrm{~N}$ in hexane, $0.5 \mathrm{ml}, 0.77 \mathrm{mmol})$ was added in drops to a stirred solution of $9(209 \mathrm{mg}, 0.77 \mathrm{mmol})$ in dry THF $(4 \mathrm{ml})$ at $-78^{\circ} \mathrm{C}$. After the solution was stirred at $-78^{\circ} \mathrm{C}$ for $30 \mathrm{~min}$, a solution of fumaraldehyde mono(dimethylacetal) $(\mathbf{1 0} ; 100 \mathrm{mg}, 0.77 \mathrm{mmol})$ in dry THF $(2 \mathrm{ml})$ was added in drops. The whole was stirred for $2 \mathrm{~h}$ at $-78^{\circ} \mathrm{C}$, and then stirred in an ice bath for $15 \mathrm{~min}$. Then, the reaction was quenched by adding an aqueous solution of $0.1 \mathrm{~N} \mathrm{HCl}(3.9 \mathrm{ml})$. The mixture was stirred again in the ice bath for $30 \mathrm{~min}$, and then at room temperature for $1 \mathrm{~h}$. Then the mixture was cooled with ice and treated with a saturated $\mathrm{NaHCO}_{3}$ solution. The 
mixture was partitioned between water and EtOAc. The organic layer was dried over $\mathrm{MgSO}_{4}$ and the solvents were removed in vacuo to afford a crystalline residue, which was recrystallized from hexane-AcOEt to afford 6j $(127 \mathrm{mg}, 82 \%)$ as a yellow solid, mp $174-177^{\circ} \mathrm{C}$. Anal. Calcd for $\mathrm{C}_{12} \mathrm{H}_{8} \mathrm{FNO}$ : C, 71.64; H, 4.01; N, 6.96. Found: C, 71.84; H, 4.27; N, 6.83 . IR $(\mathrm{KBr}) \mathrm{cm}^{-1}: 2230,1681,1672,1621,1421,1159,1124 .{ }^{1} \mathrm{H}-\mathrm{NMR}$ $\left(270 \mathrm{MHz}, \mathrm{CDCl}_{3}\right) \delta: 6.36(1 \mathrm{H}, \mathrm{dd}, J=15,8 \mathrm{~Hz}), 7.14(1 \mathrm{H}, \mathrm{d}-\mathrm{like}, J=3 \mathrm{~Hz})$, $7.16(1 \mathrm{H}, \mathrm{d}, J=8 \mathrm{~Hz}), 7.28(1 \mathrm{H}$, ddd, $J=15,8,3 \mathrm{~Hz}), 7.40(1 \mathrm{H}, \mathrm{dd}, J=10,1$ $\mathrm{Hz}), 7.47(1 \mathrm{H}, \mathrm{dd}, J=8,1 \mathrm{~Hz}), 7.67(1 \mathrm{H}, \mathrm{t}, J=8 \mathrm{~Hz}), 9.68(1 \mathrm{H}, \mathrm{d}, J=8 \mathrm{~Hz})$. MS $m / z 201\left(\mathrm{M}^{+}\right), 172(100 \%), 158,145$.

4-[(1E,3E)-4-[trans-5-[I(1R,2R)-2-(2,4-Difluorophenyl)-2-hydroxy-1methyl-3-(1H-1,2,4-triazol-1-yl)propyl]thio]-1,3-dioxan-2-yl]-1,3-butadienyl]-3-fluorobenzonitrile (CS-758, 3j) A mixture of $\mathbf{4}(8.73 \mathrm{~g}, 24.3$ $\mathrm{mmol}), \mathbf{6 j}(4.63 \mathrm{~g}, 23.0 \mathrm{mmol})$ and $p$-toluenesulfonic acid monohydrate $(5.07 \mathrm{~g}, 26.7 \mathrm{mmol})$ in dry THF $(200 \mathrm{ml})$ was allowed to stand at room temperature for $30 \mathrm{~min}$. Then the solvent was removed using a rotary evaporator. The residue was suctioned using a vacuum pump at room temperature to afford an oily residue. Similar evaporation cycles (addition of THF, allowing the mixture to stand, and evaporation) were repeated two more times. To the residue, THF $(150 \mathrm{ml})$ was added, and the solution was poured slowly into a stirred aqueous solution of saturated $\mathrm{NaHCO}_{3}$ at $0^{\circ} \mathrm{C}$. The product was extracted with EtOAc. The organic layer was washed with brine. The extract was dried over $\mathrm{MgSO}_{4}$ and solvents were removed in vacuo to afford a pale yellow oil, which was chromatographed on silica gel $(500 \mathrm{~g}$, hexane-AcOEt, $2: 1, \mathrm{v} / \mathrm{v})$ to afford CS-758 (3j) $(9.35 \mathrm{~g}, 74 \%)$ as a yellow amorphous solid. An analytical sample, mp $127-128^{\circ} \mathrm{C}$, was obtained by recrystallization from EtOAc-hexane. $[\alpha]_{\mathrm{D}}^{25}-78.3^{\circ}\left(c=1.8, \mathrm{CHCl}_{3}\right)$. Anal. Calcd for $\mathrm{C}_{27} \mathrm{H}_{25} \mathrm{~F}_{3} \mathrm{~N}_{4} \mathrm{O}_{3} \mathrm{~S}$ : C, 59.77; H, 4.64; F, 10.50; N, 10.33; S, 5.91. Found: C, 59.66; H, 4.62; F, 10.38; N, 10.24; S, 5.92. IR (KBr) cm $\mathrm{cm}^{-1}: 2232,1616$, $1499,1140 .{ }^{1} \mathrm{H}-\mathrm{NMR}\left(400 \mathrm{MHz}, \mathrm{CDCl}_{3}\right) \delta: 1.19(3 \mathrm{H}, \mathrm{d}, J=7 \mathrm{~Hz}), 3.33(1 \mathrm{H}$, q, $J=7 \mathrm{~Hz}), 3.40(1 \mathrm{H}, \mathrm{tt}, J=11,5 \mathrm{~Hz}), 3.62(1 \mathrm{H}, \mathrm{t}, J=11 \mathrm{~Hz}), 3.64(1 \mathrm{H}, \mathrm{t}$, $J=11 \mathrm{~Hz}), 4.30(1 \mathrm{H}, \mathrm{ddd}, J=11,5,2 \mathrm{~Hz}), 4.43(1 \mathrm{H}, \mathrm{ddd}, J=11,5,2 \mathrm{~Hz})$, $4.83(1 \mathrm{H}, \mathrm{d}, J=14 \mathrm{~Hz}), 5.01(1 \mathrm{H}, \mathrm{s}), 5.03(1 \mathrm{H}, \mathrm{d}, J=14 \mathrm{~Hz}), 5.07(1 \mathrm{H}, \mathrm{d}$, $J=4 \mathrm{~Hz}), 5.90(1 \mathrm{H}, \mathrm{dd}, J=15,4 \mathrm{~Hz}), 6.62(1 \mathrm{H}, \mathrm{dd}, J=15,11 \mathrm{~Hz}), 6.7-6.8$ $(2 \mathrm{H}, \mathrm{m}), 6.73(1 \mathrm{H}, \mathrm{d}, J=16 \mathrm{~Hz}), 6.95(1 \mathrm{H}, \mathrm{dd}, J=16,11 \mathrm{~Hz}), 7.3-7.4(1 \mathrm{H}$, $\mathrm{m}), 7.34(1 \mathrm{H}, \mathrm{d}, J=9 \mathrm{~Hz}), 7.40(1 \mathrm{H}, \mathrm{d}, J=8 \mathrm{~Hz}), 7.58(1 \mathrm{H}, \mathrm{t}, J=8 \mathrm{~Hz}), 7.79$ $(2 \mathrm{H}, \mathrm{s})$. MS (FAB) $m / z 543(\mathrm{M}+\mathrm{H})^{+}$.

Determination of MICs MICs were determined by the broth microdilution method according to the guidelines in National Committee for Clinical Laboratory Standard (NCCLS) documents 1997 M27-A, 1995 M27-T, and 1998 M38-P, Wayne, PA. Roswell Park Memorial Institute (RPMI) 1640 medium (GIBCO) (for C. neoformans: yeast nitrogen base) buffered with 0.165 м 3-(morpholino)propanesulfonic acid (MOPS) ( $\mathrm{pH} 7.0$ ) was used. Microplates (96 wells) were inoculated at a concentration of $1-5 \times 10^{3}$ cells $/ \mathrm{ml}$ for Candida species, ca. $1 \times 10^{4}$ cells $/ \mathrm{ml}$ for C. neoformans, and $2.5 \times 10^{4}$ spores $/ \mathrm{ml}$ for Aspergillus species, and incubated at $35^{\circ} \mathrm{C}$ (for $A s-$ pergillus species: $30^{\circ} \mathrm{C}$ ). The MICs were read when obvious growth was observed in compound-free control wells. The MICs were defined as the lowest compound concentrations causing at least $80 \%$ growth inhibition compared with the control.

Acknowlegement We acknowledge the contribution of Dr. Yasuhiro Ito of Planning \& Coordination Department of our company for the coordination of the project and active discussions.

\section{References and Notes}

1) Oida S., Tajima Y., Konosu T., Nakamura Y., Somada A., Tanaka T., Habuki S., Harasaki T., Kamai Y., Fukuoka T., Ohya S., Yasuda H., Chem. Pharm. Bull., 48, 694-707 (2000).

2) The elimination half-life of R-102557 in plasma was $37.4 \mathrm{~h}$ in rats.

3) A part of this work was presented by the authors at the 40th Interscience Conference on Antimicrobial Agents and Chemotherapy, American Society for Microbiology, Poster No. 1087, Sep. 17-20, 2000, Toronto.

4) We did not investigate derivatives with longer ( $n \geqq 3)$ side chains because their syntheses would require more steps. A separate experiment we carried out showed that a compound having three double bonds $(n=3)$ with a 4-(trifluoromethyl)phenyl group $\left(\mathrm{Ar}=4-\left(\mathrm{CF}_{3}\right) \mathrm{C}_{6} \mathrm{H}_{4}\right)$ had lower activities than $\mathbf{3 b}(n=2)$ in vivo in mice (unpublished data).

5) The effects of the fluorine atom can be seen in the results of the preliminary experiments. The concentration of CS-758 reached $13 \mu \mathrm{g} / \mathrm{ml}$ when a capsule (No. 2) containing CS-758 $(50 \mathrm{mg})$ and polysorbate 80 $(300 \mathrm{mg})$ was stirred in a JP-2 solution $(900 \mathrm{ml})$ at $37^{\circ} \mathrm{C}$. For $\mathbf{4 f}$, the maximum concentration was $4-5 \mu \mathrm{g} / \mathrm{ml}$. The half-life of CS-758 in $\mathrm{HCl}(0.007 \mathrm{~mol} / \mathrm{l})$ solution in $\mathrm{CH}_{3} \mathrm{CN}-\mathrm{H}_{2} \mathrm{O}(3: 7, \mathrm{v} / \mathrm{v})$ at $37^{\circ} \mathrm{C}$ was $6.40 \mathrm{~min}$, whereas that of $\mathbf{3 f}$ was $3.12 \mathrm{~min}$. The absolute bioavailability (BA) of CS-758 in rats after oral administration $(20 \mathrm{mg} / \mathrm{kg})$ of its poly(ethylene glycol) 400 solution was $113 \%$, whereas that of $\mathbf{3 f}$ was $50.7 \%$. The elimination half-life of CS-758 in plasma was $9.1 \mathrm{~h}$ in rats, whereas that of $\mathbf{3 f}$ was $5.7 \mathrm{~h}$.

6) Xue C.-B., Wityak J., Sielecki T. M., Pinto. D. J., Batt D. G., Cain G. A., Sworin M., Rockwell A. L., Roderick J. J., Wang S., Orwat M. J., Frietze W. E., Bostrom L. L., Liu J., Higley C. A., Rankin F. W., Tobin A. E., Emmett G., Lalka G. K., Sze J. Y., Meo S. V. D., Mousa S. A., Thoolen M. J., Racanelli A. L., Hausner E. A., Reilly T. M., Degrado W. F., Wexler R. R., Olson R. E., J. Med. Chem., 40, 2064-2084 (1997); Lee K., Jung W.-H., Hwang S. Y., Lee S.-H., Bioorg. Med. Chem. Lett., 9, 2483-2486 (1999). 